\title{
Limit cycles in a quartic system with a third-order nilpotent singular point
}

\section{Xinli Li*i}

\section{"Correspondence:}

lixinli0539@sina.com

School of Business, University of

Shanghai for Science and

Technology, Shanghai, P.R. China

Logistic School, Linyi University,

Linyi, P.R. China

\begin{abstract}
In this paper, limit cycles bifurcating from a third-order nilpotent critical point in a class of quartic planar systems are studied. With the aid of computer algebra system MAPLE, the first 12 Lyapunov constants are deduced by the normal form method. As a result, sufficient and necessary center conditions are derived, and the fact that there exist 12 or 13 limit cycles bifurcating from the nilpotent critical point is proved by different perturbations. The result in [Qiu et al. in Adv. Differ. Equ. 2015(1):1, 2015] is improved.
\end{abstract}

Keywords: Quartic system; Nilpotent critical point; Lyapunov constants; Bifurcation of limit cycles

\section{Introduction}

An isolated critical point $O(0,0)$ is called a nilpotent singular point if the linear part of systems has double zero eigenvalues but the matrixes of the linearized systems at the origin are not identically null. Over the past twenty years, many different kinds of topological phase portraits around a nilpotent critical point have been given in [2]. Some results before 1980 were also introduced in [3]. It is well known that it is more difficult to study a phase portrait around a nilpotent critical point than around an element critical point. However, more and more attention has been paid to the center problem and bifurcation of limit cycles of a system with a nilpotent critical point recently.

By a proper linear transformation, the planar autonomous analytic system with a nilpotent critical point can always be changed into

$$
\begin{aligned}
& \frac{d x}{d t}=\Phi(x, y)=y+\sum_{k+j=2}^{\infty} a_{k j} x^{k} y^{j}, \\
& \frac{d y}{d t}=\Psi(x, y)=\sum_{k+j=2}^{\infty} b_{k j} x^{k} y^{j},
\end{aligned}
$$

where $\Phi(x, y)$ and $\Psi(x, y)$ are analytic functions in the neighborhood of origin.

\section{Springer}


On the basis of the discussion in [3], the origin of system (1.1) is a monodromic critical point if and only if

$$
\begin{aligned}
& \Psi\left(x, f(x)=\alpha x^{2 n-1}+o\left(x^{2 n-1}\right), \quad \alpha \neq 0,\right. \\
& {\left[\frac{\partial \Phi}{\partial x}+\frac{\partial \Psi}{\partial y}\right]_{y=f(x)}=\beta x^{n-1}+o\left(x^{n-1}\right),} \\
& \beta^{2}+4 n \alpha<0,
\end{aligned}
$$

where $n$ is a positive integer. The origin is called a $(2 n-1)$-order nilpotent critical point.

In [4], local behavior of an isolated nilpotent critical point for polynomial Hamiltonian systems was investigated. They proved that there are exactly three cases: a center, a cusp, or a saddle. Then, for quadratic and cubic Hamiltonian systems, they obtained necessary and sufficient conditions for a nilpotent critical point to be a center, a cusp, or a saddle. Some special systems were also studied in $[5,6]$ by this method. Furthermore, limit cycle bifurcation near a double homoclinic loop passing through a nilpotent saddle by studying the analytical property of the first-order Melnikov functions for general near-Hamiltonian systems was studied in [7]. They obtained the conditions for the perturbed system to have 8,10 , or 12 limit cycles in a neighborhood of the loop with seven different distributions. Recently, the expansion of the first Melnikov function appearing by perturbing an integrable and reversible system with a homoclinic loop passing through a nilpotent singular point was investigated in [8], and the authors got the formulas for computing the first coefficients of the expansion. Some other methods were also developed recently. For example, in [9], it was proved that all the nilpotent centers are limit of linear type centers; and consequently, the Poincare-Lyapunov method to find linear type centers can be also used to find the nilpotent centers. Quasi-homogeneous vector fields with a nilpotent and monodromic isolated singular point were investigated in [10]. They also proved for this system the existence of a Lyapunov function, and they solved theoretically the center problem for such a system. Bifurcation theory for finitely smooth planar autonomous differential systems was considered in [11]. Theory of rotated equations was discussed in [12] and was applied to a population model.

The origin is called a third-order nilpotent critical point when $n=2$ in (1.2). For the planar systems with a third-order nilpotent critical point, the center problem has been completely solved in [13] where the author proved that the system has always an integrating factor, but this method is not valid for any nilpotent singularity. The inverse integrating factor method for the planar systems with a third-order nilpotent critical point was also discussed in $[14,15]$. By using those methods, the fact that there exist eight small amplitude limit cycles created from nilpotent critical points was proved in [16] for a class of cubic systems by their method. Furthermore, a new kind of bifurcation phenomenon was discussed in [17]. They proved that a third-order nilpotent focus of the planar dynamical systems could be broken into two element foci and an element saddle, limit cycles could bifurcate out from two-element focus. As an example, a class of cubic systems with 3-multiple nilpotent focus were investigated. They proved that nine limit cycles could bifurcate from the origin when the origin is a weak focus of order eight. For third-order nilpotent critical points of a planar dynamical system, the analytic center problem was completely solved by using the integrating factor method [18]. By using this method, some special systems were investigated in $[1,19,20]$. 
In fact, Theorem 19.10 in [3] shows when condition (1.2) holds, there exist formal transformations

$$
\begin{aligned}
& u=x+\sum_{k+j=2}^{\infty} a_{k j}^{\prime} x^{k} y^{j}, \\
& v=y+\sum_{k+j=2}^{\infty} b_{k j}^{\prime} x^{k} y^{j}, \\
& \frac{d t}{d \tau}=1+\sum_{k+j=1}^{\infty} c_{k j}^{\prime} x^{k} y^{j},
\end{aligned}
$$

such that (1.1) becomes the Liénard equation

$$
\frac{d u}{d \tau}=v+F(u), \quad \frac{d v}{d \tau}=\alpha u^{2 n-1},
$$

where

$$
F(u)=\frac{1}{n} \beta u^{n}+o\left(u^{n}\right)
$$

Furthermore, if condition (1.2) holds, there exist analytic transformations in the neighborhood of origin of (1.3) to make (1.1) be changed into the Liénard equation

$$
\frac{d u}{d \tau}=v, \quad \frac{d v}{d \tau}=\alpha u^{2 n-1}+v \sum_{k=n-1}^{\infty} B_{k} u^{k}, \quad B_{n-1}=\beta,
$$

so $B_{2 k}$ could be thought of as Lyapunov constants of (1.1).

From then, the normal form theory was applied to solve the center-focus problem for monodromic planar nilpotent singularities. In [3,21-24], by considering the normal forms of (1.1), the authors tried to study the computation problem of focal values. In [25], by means of a recursive algorithm well suited to symbolic computation of normal form, authors achieved the expressions for its coefficients and investigated the possibilities of simplifying the classical normal form, obtaining simpler and higher order normal forms than in previous works. Furthermore, in [26], authors showed that normal form can also be applied to generate limit cycles from nilpotent singularities. In [27], Hamiltonian linear type centers and nilpotent centers of linear plus cubic polynomial vector fields were considered. They provided twelve normal forms for all the Hamiltonian planar polynomial vector fields having linear plus cubic homogeneous terms which possess a linear type center or a nilpotent center at the origin, and found their global phase portraits on the Poincare disk. Last year, the normal form method was used to investigated the nilpotent critical point again by Pei Yu. The authors gave an efficient program by MAPLE in [28]. Ten limit cycles bifurcating from a nilpotent critical point in a cubic system are obtained. For a quartic system with third-order critical point, 11 limit cycles were obtained in [1] by using the inverse integrating factor method. In this paper, a class of quartic systems with 
third-order nilpotent singular point

$$
\begin{aligned}
\frac{d x}{d t}= & y-b_{12} x^{2} y+a_{12} x y^{2}+a_{03} y^{3}+a_{40} x^{4}+a_{31} x^{3} y+a_{22} x^{2} y^{2}-4 b_{04} x y^{3}+a_{04} y^{4} \\
\frac{d y}{d t}= & -2 x^{3}+b_{21} x^{2} y+b_{12} x y^{2}+b_{03} y^{3}+b_{40} x^{4}-4 a_{40} x^{3} y-\frac{3}{2} a_{31} x^{2} y^{2} \\
& +b_{13} x y^{3}+b_{04} y^{4}
\end{aligned}
$$

are studied by using the normal form method, one more limit cycle is found.

The rest of this paper is organized as follows. In Sect. 2, with the aid of computer algebra system MAPLE, the first 12 Lyapunov constants are computed by the normal form method. Sufficient and necessary center conditions are derived. In Sect. 3, the fact that there exist 12 or 13 limit cycles bifurcating from the nilpotent critical point is proved. Henceforth, we give a lower bound of cyclicity of a nilpotent critical point for quartic polynomial systems. The result in [1] is improved.

\section{Lyapunov constants and center conditions}

According to normal form theory, for system (1.1), we can find analytic transformations in the neighborhood of origin to transform system (1.7) into (1.6). Applying the recursive formulae in [28] to carry out calculations, we have the following theorem.

Theorem 2.1 The first 12 quasi-Lyapunov constants at the origin of system (1.7) are given as follows:

$$
\begin{aligned}
B_{2}= & \frac{b_{21}}{3}, \\
B_{4}= & \frac{2\left(a_{12}+3 b_{03}\right)}{5}, \\
B_{6}= & \frac{b_{40}\left(2 a_{22}+3 b_{13}\right)}{35}, \\
B_{8}= & -\frac{\left(2 a_{22}+3 b_{13}\right) a_{31}}{15}, \\
B_{10}= & \frac{10}{77}\left(3 a_{40} b_{03}-2 b_{04}\right)\left(2 a_{22}+3 b_{13}\right), \\
B_{12}= & \frac{-2}{3003} b_{03}\left(342 a_{22}+539 a_{40} b_{12}-26 b_{13}\right)\left(2 a_{22}+3 b_{13}\right), \\
B_{14}= & -\frac{1}{83,655} b_{03}\left(36,504 a_{04}+17,745 a_{40}^{3}-20,744 a_{22} b_{12}-31,116 a_{40} b_{12}^{2}\right) \\
& \left(2 a_{22}+3 b_{13}\right), \\
B_{16}= & \frac{14}{1,680,705} b_{03}\left(217,464 a_{03} a_{22}+398,853 a_{22} a_{40}^{2}+334,620 a_{40} b_{03}^{2}\right. \\
& \left.+326,196 a_{03} a_{40} b_{12}+561,522 a_{40}^{3} b_{12}+12,244 a_{22} b_{12}^{2}+18,366 a_{40} b_{12}^{3}\right) \\
& \times\left(2 a_{22}+3 b_{13}\right) .
\end{aligned}
$$


Case 1: $2 a_{22}+3 a_{40} b_{12} \neq 0$

$$
\begin{aligned}
B_{18}= & \frac{b_{03}\left(2 a_{22}+3 b_{13}\right)}{133,545,457,890\left(2 a_{22}+3 a_{40} b_{12}\right)}\left(409,339,646,784 a_{22}^{3} a_{40}\right. \\
& -76,483,842,435 a_{22} a_{40}^{5} \\
& +265,048,602,624 a_{22}^{2} b_{03}^{2}+100,943,142,300 a_{40}^{4} b_{03}^{2} \\
& +2,055,888,104,472 a_{22}^{2} a_{40}^{2} b_{12} \\
& -125,814,214,890 a_{40}^{6} b_{12}+1,353,439,930,752 a_{22} a_{40} b_{03}^{2} b_{12} \\
& +3,384,047,982,384 a_{22} a_{40}^{3} b_{12}^{2}+1,433,800,540,224 a_{40}^{2} b_{03}^{2} b_{12}^{2} \\
& -3,536,917,504 a_{22}^{2} b_{12}^{3}+1,831,845,046,410 a_{40}^{4} b_{12}^{3} \\
& \left.-10,610,752,512 a_{22} a_{40} b_{12}^{4}-7,958,064,384 a_{40}^{2} b_{12}^{5}\right), \\
B_{20}= & -b_{03}\left(2 a_{22}+3 b_{13}\right) f_{1}, \\
B_{22}= & b_{03}\left(2 a_{22}+3 b_{13}\right) f_{2}, \\
B_{24}= & b_{03}\left(2 a_{22}+3 b_{13}\right) f_{3} .
\end{aligned}
$$

Case 2: $2 a_{22}+3 a_{40} b_{12}=0$

$$
\begin{aligned}
& B_{16}=\frac{7}{663} a_{40} b_{03}\left(264 b_{03}^{2}-29 a_{40}^{2} b_{12}\right)\left(2 a_{22}+3 b_{13}\right), \\
& B_{18}=-\frac{1}{627} a_{40}^{3} b_{03}\left(154 a_{03}+462 a_{40}^{2}-87 b_{12}^{2}\right)\left(2 a_{22}+3 b_{13}\right), \\
& B_{20}=\frac{1}{70,686} a_{40}^{3} b_{03} b_{12}\left(9779 a_{40}^{2}-1740 b_{12}^{2}\right)\left(2 a_{22}+3 b_{13}\right), \\
& B_{22}=\frac{4,589,527}{6,273,135} a_{40}^{7} b_{03}\left(2 a_{22}+3 b_{13}\right) .
\end{aligned}
$$

For each $k=2, \ldots, 11, B_{2}=B_{4}=\cdots=B_{2 k-2}=0$ have been set. $f_{i}, i=1,2,3$, are given in the Appendix. They have 26, 30, 48 terms, respectively.

Theorem 2.1 directly shows the following assertion.

Proposition 2.1 For system (1.7), the first twelve Lyapunov constants at the origin are zero if and only if one of the following three conditions is satisfied:

$$
\begin{aligned}
& b_{21}=a_{12}=b_{03}=b_{40}=a_{31}=b_{04}=0 ; \\
& b_{21}=0, \quad a_{12}=-3 b_{03}, \quad a_{22}=-\frac{3 b_{13}}{2} ; \\
& b_{21}=a_{31}=b_{40}=b_{04}=b_{13}=a_{04}=a_{22}=a_{40}=0, \quad a_{12}=-3 b_{03} .
\end{aligned}
$$

According to Proposition 2.1, we have the following theorem.

Theorem 2.2 The origin of system (1.7) is a center if and only if the first twelve Lyapunov constants are zero, that is, one of the conditions in Proposition 2.1 is satisfied. 
Figure 1 Phase portrait of system (2.7)

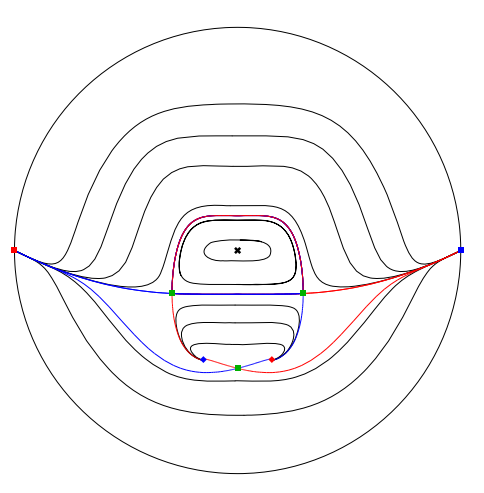

Proof When condition (2.4) is satisfied, system (1.7) can be brought to

$$
\begin{aligned}
& \frac{d x}{d t}=y-b_{12} x^{2} y+a_{03} y^{3}+a_{40} x^{4}+a_{22} x^{2} y^{2}+a_{04} y^{4} \\
& \frac{d y}{d t}=-2 x^{3}+b_{12} x y^{2}-4 a_{40} x^{3} y+b_{13} x y^{3}
\end{aligned}
$$

whose vector field is symmetric with respect to the $y$-axis. An example when all parameters are equal to 1 is given in Fig. 1 .

When condition (2.5) holds, system (1.7) can be rewritten as follows:

$$
\begin{aligned}
\frac{d x}{d t}= & y-b_{12} x^{2} y-3 b_{03} x y^{2}+a_{03} y^{3}+a_{40} x^{4}+a_{31} x^{3} y \\
& -\frac{3}{2} b_{13} x^{2} y^{2}-4 b_{04} x y^{3}+a_{04} y^{4} \\
\frac{d y}{d t}= & -2 x^{3}+b_{12} x y^{2}+b_{03} y^{3}+b_{40} x^{4}-4 a_{40} x^{3} y-\frac{3}{2} a_{31} x^{2} y^{2}+b_{13} x y^{3}+b_{04} y^{4},
\end{aligned}
$$

which has an analytic first integral

$$
\begin{aligned}
H_{1}(x, y)= & \frac{1}{2} y^{2}+\frac{1}{2} x^{4}+\left(\frac{a_{03}}{4} y^{4}+\frac{a_{04}}{5} y^{5}-\frac{b_{40}}{5} x^{5}-\frac{b_{12}}{2} x^{2} y^{2}\right. \\
& \left.-\frac{a_{31}}{2} x^{3} y^{2}-b_{03} x y^{3}-\frac{b_{13}}{2} x^{2} y^{3}-b_{04} x y^{4}\right) .
\end{aligned}
$$

An example when all parameters are equal to 1 is given in Fig. 2.

When condition (2.6) holds, system (1.7) becomes

$$
\begin{aligned}
& \frac{d x}{d t}=y-b_{12} x^{2} y-3 b_{03} x y^{2}+a_{03} y^{3} \\
& \frac{d y}{d t}=-2 x^{3}+b_{12} x y^{2}+b_{03} y^{3}
\end{aligned}
$$

which has an analytic first integral

$$
H_{2}(x, y)=\frac{1}{2} y^{2}+\frac{1}{2} x^{4}-\left(\frac{b_{12}}{2} x^{2} y^{2}+b_{03} x y^{3}\right) \text {. }
$$

An example when all parameters are equal to 1 is given in Fig. 3. 
Figure 2 Phase portrait of system (2.8)

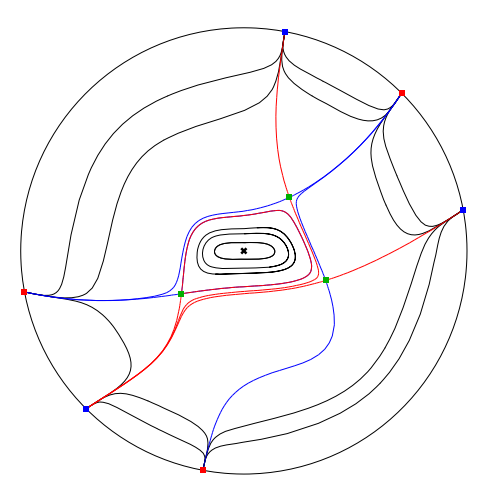

Figure 3 Phase portrait of system (2.10)

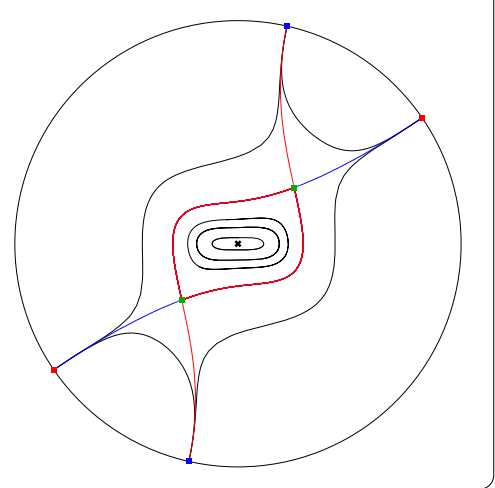

Remark 2.1 The center condition of system (1.7) is the same as the center condition of system in [1].

\section{Bifurcation of a limit cycle from the origin of system (1.7)}

In this section, two perturbation methods are given in order to obtain more limit cycles. The first method is called perturbation method of small parameters by perturbing the coefficients. The second method is double bifurcation.

\subsection{Perturbation method of small parameters}

At first, we will prove that the perturbed system of (1.7) can generate twelve limit cycles enclosing an elementary node at the origin of unperturbed system (1.7) when the third-order nilpotent critical point $O(0,0)$ is a 12 th-order weak focus by perturbing the coefficients.

$B_{2}=B_{4}=B_{6}=B_{8}=B_{10}=B_{12}=B_{14}=B_{16}=B_{18}=B_{20}=B_{22}=0,=B_{24} \neq 0$ show the following.

Theorem 3.1 For system (1.7), the origin is a 12th-order weak focus if and only if $a_{22} b_{03} b_{12}\left(2 a_{22}+3 b_{13}\right) \neq 0$ and

$$
\begin{aligned}
& b_{21}=b_{40}=a_{31}=0, \\
& a_{12}=-3 b_{03}, \\
& b_{04}=\frac{3 a_{40} b_{03}}{2},
\end{aligned}
$$




$$
\begin{aligned}
b_{13}= & \frac{342 a_{22}+539 a_{40} b_{12}}{26}, \\
a_{04}= & -\frac{28,665 a_{40}^{3}+56,568 a 22 b_{12}+91,700 a_{40} b_{12}^{2}-6848 b_{12} b_{13}}{58,968}, \\
a_{03}= & -\left(398,853 a 22 a_{40}^{2}+334,620 a_{40} b 03^{2}+561,522 a_{40}^{3} b_{12}+12,244 a 22 b_{12}^{2}\right. \\
& \left.+18,366 a_{40} b_{12}^{3}\right) /\left(108,732\left(2 a_{22}+3 a_{40} b_{12}\right)\right), \\
b_{03}^{2}= & f_{4} /\left(4 6 8 \left(566,343,168 a_{22}^{2}+215,690,475 a_{40}^{4}+2,891,965,664 a_{22} a_{40} b_{12}\right.\right. \\
& \left.\left.+3,063,676,368 a_{40}^{2} b_{12}^{2}\right)\right),
\end{aligned}
$$

where

$$
\begin{aligned}
f_{4}= & -409,339,646,784 a_{22}^{3} a_{40}+76,483,842,435 a_{22} a_{40}^{5} \\
& -2,055,888,104,472 a_{22}^{2} a_{40}^{2} b_{12} \\
& +125,814,214,890 a_{40}^{6} b_{12}-3,384,047,982,384 a_{22} a_{40}^{3} b_{12}^{2} \\
& +3,536,917,504 a_{22}^{2} b_{12}^{3} \\
& -1,831,845,046,410 a_{40}^{4} b_{12}^{3}+10,610,752,512 a_{22} a_{40} b_{12}^{4} \\
& +7,958,064,384 a_{40}^{2} b_{12}^{5} .
\end{aligned}
$$

Proof From $B_{2}=B_{4}=B_{6}=B_{8}=B_{10}=B_{12}=B_{14}=B_{16}=B_{18}=0$, we obtain

$$
\begin{aligned}
b_{21}= & b_{40}=a_{31}=0, \\
a_{12}= & -3 b_{03}, \quad b_{04}=\frac{3 a_{40} b_{03}}{2}, \\
b_{13}= & \frac{342 a_{22}+539 a_{40} b_{12}}{26}, \\
a_{04}= & -\frac{28,665 a_{40}^{3}+56,568 a_{22} b_{12}+91,700 a_{40} b_{12}^{2}-6848 b_{12} b_{13}}{58,968}, \\
a_{03}= & -\left(398,853 a_{22} a_{40}^{2}+334,620 a_{40} b 03^{2}+561,522 a_{40}^{3} b_{12}+12,244 a_{22} b_{12}^{2}\right. \\
& \left.+18,366 a_{40} b_{12}^{3}\right) /\left(108,732\left(2 a_{22}+3 a_{40} b_{12}\right)\right), \\
b_{03}^{2}= & f_{4} /\left(4 6 8 \left(566,343,168 a_{22}^{2}+215,690,475 a_{40}^{4}+2,891,965,664 a_{22} a_{40} b_{12}\right.\right. \\
& \left.\left.+3,063,676,368 a_{40}^{2} b_{12}^{2}\right)\right),
\end{aligned}
$$

and let

$$
f_{12}=\operatorname{Res}\left[f_{1}, f_{2}, a_{22}\right], \quad f_{13}=\operatorname{Res}\left[f_{1}, f_{3}, a_{22}\right],
$$

$f_{12}$ and $f_{13}$ have 20,23 terms, respectively.

$$
\operatorname{Res}\left[f_{12}, f_{13}, b_{12}\right]=6.48339335810581 \times 10^{15,074} a_{40}^{1672} .
$$

If $a_{40}=0$, we get $a_{22}=0$, which contradicts $2 a_{22}+3 a_{40} b_{12} \neq 0$. So, when condition (3.3) holds, the origin of system (1.7) is a 12 th-order weak focus. 
Next, we study the perturbed system of (1.7) given by

$$
\begin{aligned}
\frac{d x}{d t}= & \delta x+y-b_{12} x^{2} y+a_{12} x y^{2}+a_{03} y^{3}+a_{40} x^{4}+a_{31} x^{3} y+a_{22} x^{2} y^{2} \\
& -4 b_{04} x y^{3}+a_{04} y^{4} \\
\frac{d y}{d t}= & \delta y-2 x^{3}+b_{21} x^{2} y+b_{12} x y^{2}+b_{03} y^{3}+b_{40} x^{4}-4 a_{40} x^{3} y \\
& -\frac{3}{2} a_{31} x^{2} y^{2}+b_{13} x y^{3}+b_{04} y^{4} .
\end{aligned}
$$

When conditions in (3.3) hold, using relationships $B_{2}=B_{4}=B_{6}=B_{8}=B_{10}=B_{12}=B_{14}=$ $B_{16}=B_{18}=B_{20}=B_{22}=0$, we can determine the values of

$$
b_{21}, a_{12}, b_{40}, a_{31}, b_{04}, b_{13}, a_{04}, a_{03}, b_{03} \text {. }
$$

Hence, when the conditions in (3.3) are satisfied, we have

$$
\begin{aligned}
& \frac{\partial\left(B_{2}, B_{4}, B_{6}, B_{8}, B_{10}, B_{12}, B_{14}, B_{16}, B_{18}, B_{20}, B_{22}\right)}{\partial\left(b_{21}, a_{12}, b_{40}, a_{31}, b_{04}, b_{13}, a_{04}, a_{03}, b_{03}, a_{22}\right)} \\
& \quad=f_{5} .
\end{aligned}
$$

Denote $f_{15}=\operatorname{Res}\left[f_{1}, f_{5}, a_{22}\right]$, it has 51 terms.

$$
\operatorname{Res}\left[f_{12}, f_{15}, b_{12}\right]=6.48339335810581 \times 10^{15,074} a_{40}^{1672} \neq 0
$$

So, when condition (3.3) holds and $f_{1}=f_{2}=0$, we can conclude that $f_{5} \neq 0$.

Further, Theorem 3.1.3 in [15] yields that if the origin of system (3.4) $\left.\right|_{\delta=\varepsilon=0}$ is a weak focus of order $m$, then, when $0<\delta, \varepsilon \ll 1$, (3.4) has at most $m$ limit cycles in a neighborhood of the origin. Namely the following theorem holds.

Theorem 3.2 If the origin of system (1.1) is a 12th-order weakfocus, for $0<\delta, \varepsilon \ll 1$, then, for system (3.4), in a small neighborhood of the origin, there exist exactly 12 small amplitude limit cycles enclosing the origin $O(0,0)$, which is an elementary node.

\subsection{Double perturbation method}

In this section, an interesting bifurcation of limit cycles, which is different from the first kind of bifurcation discussed in previous section, will be considered. One more smallamplitude limit cycles near the origin can be found. Consider the following perturbed system of (1.1):

$$
\begin{aligned}
\frac{d x}{d t}= & a_{40} x^{2}\left(x^{2}-\varepsilon^{2}\right)+y\left(1+x\left(-b_{12} x+a_{31} x^{2}\right)\right)+\left(a_{12} x+a_{03} y+a_{22} x^{2}\right. \\
& \left.-4 b_{04} x y+a_{04} y^{2}\right) y^{2} \\
\frac{d y}{d t}= & \left(4 \delta \varepsilon-2 a_{40} \varepsilon^{2} x\right) y-\left(x^{2}-\varepsilon^{2}\right)\left(2 x\left(1-\frac{b_{40}}{2} x\right)-\left(b_{21}-4 a_{40} x\right) y\right) \\
& +\left(b_{12} x+b_{03} y-\frac{3}{2} a_{31} x^{2}+b_{13} x y+b_{04} y^{2}\right) y^{2}
\end{aligned}
$$


Figure 4 Phase portrait of system (3.6)

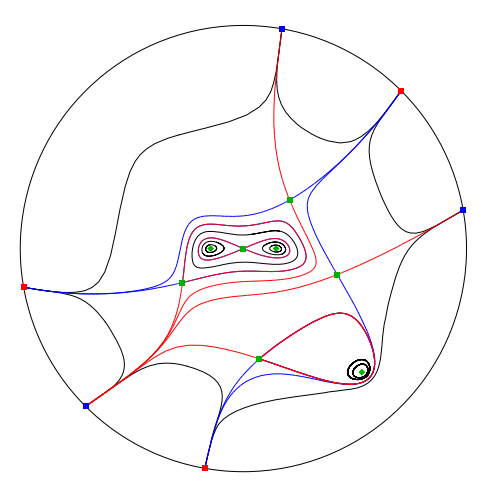

System (3.6) is called double perturbed system of system (1.7). When $0<|\varepsilon| \ll 1$, system (3.6) has three real singular points in the neighborhood of the origin, namely $O(0,0)$ and $P_{1,2}( \pm \varepsilon ; 0)$.

By transformation

$$
\begin{aligned}
& x=\varepsilon(u \pm 1), \\
& y=2 \varepsilon^{2} \frac{\left(\delta \pm a_{40} \varepsilon^{2} u\right)-\rho v}{1 \pm \varepsilon\left(-b_{12} \varepsilon+a_{31} \varepsilon^{2}\right)}, \\
& t=\frac{\tau}{2 \rho \varepsilon}, \\
& \rho=\sqrt{\left(1 \pm \varepsilon\left(\mp b_{12} \varepsilon+a_{31} \varepsilon^{2}\right)\right)\left(1 \mp \frac{b_{40}}{2} \varepsilon\right)-\left(\delta \mp a_{40} \varepsilon^{2}\right)^{2}},
\end{aligned}
$$

$P_{1,2}( \pm \varepsilon, 0)$ of system (3.6) can be shifted to the origin, and we obtain a new system in the form of

$$
\begin{aligned}
& \frac{d \xi}{d \tau}=\Phi(\xi, \eta, \varepsilon, \delta)=\frac{\delta \xi}{\rho}-\eta+\sum_{k+j=2}^{\infty} A_{k j}(\varepsilon, \delta) \xi^{k} \eta^{j}, \\
& \frac{d \eta}{d \tau}=\Psi(\xi, \eta, \varepsilon, \delta)=\xi+\frac{\delta \eta}{\rho}+\sum_{k+j=2}^{\infty} B_{k j}(\varepsilon, \delta) \xi^{k} \eta^{j},
\end{aligned}
$$

where $\Phi(\xi, \eta, \varepsilon, \delta)$ and $\Psi(\xi, \eta, \varepsilon, \delta)$ are power series in $(u, v, \varepsilon, \delta)$ with nonzero convergence radius. So $P_{1,2}( \pm \varepsilon, 0)$ of (3.6) are fine foci when $\delta \neq 0$, and weak foci or centers when $\delta=0$.

When the origin of system (1.7) is a 12th-order weak focus, the first Lyapunov constant of system (3.7) at origin is

$$
V_{1}=-\frac{1}{4} b_{21} \varepsilon+o(\varepsilon) \neq 0
$$

when $\varepsilon \rightarrow 0$. So it leads to bifurcations of two small-amplitude limit cycles around the two symmetric singular points $P_{1,2}( \pm \varepsilon, 0)$ of (3.6). Similarly, summarizing the above results yields the following theorem.

Theorem 3.3 If the origin of system (1.1) is a 12-order weak focus, choosing proper coeffcients in system (1.1), when $0<|\varepsilon| \ll 1$, there exist thirteen limit cycles with the distribution 
of one limit cycle enclosing each of $P_{1,2}( \pm \varepsilon, 0)$, and eleven limit cycles enclosing both $(\varepsilon, 0)$ and $(-\varepsilon, 0)$ in the neighborhood of origin.

So, an example which can generate 13 limit cycles by perturbing the quartic system with a nilpotent critical point is given in this section. The following result is easy to obtain from the above discussion.

Theorem 3.4 If $\delta=0, b_{21}=0, a_{12}=-3 b_{03}, a_{22}=-\frac{3 b_{13}}{2}$, there exist two centers $P( \pm \varepsilon, 0)$ and a saddle $(0,0)$ in $(3.6)$.

The Phase portrait of system (3.6) with two centers $P( \pm \varepsilon, 0)$ and a saddle $(0,0)$ was given in Fig. 4.

\section{Conclusion}

Let $M(n)$ denote the maximal number of small-amplitude limit cycles bifurcating from either an elementary focus or a center. There have been many results in the literature. $M(2)=3$ was obtained by Bautin in 1952 [29]. For $n=3$, the existence of 11 smallamplitude limit cycles around a singular point was proved by [30], which is the best result so far for cubic systems, namely $M(3)=11$. For a quartic system, using the techniques developed in [31], 21 limit cycles around an elementary focus or a center were found in [32]. For the result to quintic systems, 26 limit cycles around an elementary focus or a center were found in [32]. The authors have improved the bounds known up to now for quartic and quintic systems. Regarding the maximal number of small-amplitude limit cycles bifurcating from a nilpotent singular point, nine limit cycles were found in [28]. In [1], 12 limit cycles were obtained for a quartic system with a nilpotent singular point. In this paper, we obtained 13 limit cycles which can be bifurcated from a third-order nilpotent singular point by the normal form method. We also check our computation by the inverse integrating factor method. The result in [1] is improved.

\section{Appendix}

$$
\begin{aligned}
f_{1}= & 232,806,990,642,173,234,650,476,969,984 a_{22}^{7} \\
& -477,044,769,321,151,747,627,094,876,160 a_{22}^{5} a_{40}^{4} \\
& -82,502,158,763,349,110,891,893,788,000 a_{22}^{3} a_{40}^{8} \\
& +13,425,916,805,077,472,438,495,878,125 a_{22} a_{40}^{1} 2 \\
& +3,626,132,661,875,872,721,780,739,145,728 a_{22}^{6} a_{40} b_{12} \\
& -4,874,662,820,265,947,537,539,453,516,800 a_{22}^{4} a_{40}^{5} b_{12} \\
& -432,703,859,977,355,762,471,434,258,500 a_{22}^{2} a_{40}^{9} b_{12} \\
& +19,056,777,587,139,908,577,974,512,500 a_{40}^{1} 3 b_{12} \\
& +23,278,576,817,946,630,221,224,920,219,648 a_{22}^{5} a_{40}^{2} b_{12}^{2} \\
& -19,532,242,491,602,183,368,669,540,915,200 a_{22}^{3} a_{40}^{6} b_{12}^{2} \\
& -752,717,192,943,025,488,830,965,062,750 a_{22} a_{40}^{1} 0 b_{12}^{2} \\
& +80,182,154,821,647,691,393,757,541,171,200 a_{22}^{4} a_{40}^{3} b_{12}^{3}
\end{aligned}
$$




$$
\begin{aligned}
& -38,225,551,446,390,544,749,673,769,232,000 a_{22}^{2} a_{40}^{7} b_{12}^{3} \\
& -433,744,350,169,117,045,973,492,828,250 a_{40}^{1} 1 b_{12}^{3} \\
& -763,796,971,844,160,152,666,112,000 a_{22}^{5} b_{12}^{4} \\
& +160,998,064,918,174,797,651,106,928,670,720 a_{22}^{3} a_{40}^{4} b_{12}^{4} \\
& -36,568,732,582,402,041,283,807,931,942,400 a_{22} a_{40}^{8} b_{12}^{4} \\
& -5,773,134,335,673,254,169,280,512,000 a_{22}^{4} a_{40} b_{12}^{5} \\
& +189,440,538,958,857,143,945,451,460,248,576 a_{22}^{2} a_{40}^{5} b_{12}^{5} \\
& -13,711,504,717,817,841,782,955,386,017,440 a_{40}^{9} b_{12}^{5} \\
& -14,167,203,178,857,126,540,705,792,000 a_{22}^{3} a_{40}^{2} b_{12}^{6} \\
& +121,457,742,579,096,223,411,904,427,643,392 a_{22} a_{40}^{6} b_{12}^{6} \\
& -11,593,248,573,008,543,300,370,432,000 a_{22}^{2} a_{40}^{3} b_{12}^{7} \\
& +32,837,212,576,741,882,904,483,077,930,752 a_{40}^{7} b_{12}^{7} \\
& +2,245,173,056,476,346,827,726,848,000 a_{22} a_{40}^{4} b_{12}^{8} \\
& +5,064,667,464,745,198,665,216,000,000 a_{40}^{5} b_{12}^{9}, \\
& f_{2}=-1,024,947,112,912,004,777,224,251,017,134,080 a_{22}^{6} a_{40}^{3} \\
& -266,474,629,928,468,613,669,557,286,240,000 a_{22}^{4} a_{40}^{7} \\
& +2,872,149,434,420,042,500,705,386,405,000 a_{22}^{2} a_{40}^{11} \\
& -445,566,812,245,372,227,872,915,578,125 a_{40}^{15} \\
& +291,969,434,543,132,541,842,261,954,002,944 a_{22}^{7} b_{12} \\
& \text { - 12,365,037,948,610,082,121,455,696,104,857,600a } a_{22}^{5} a_{40}^{4} b_{12} \\
& -1,807,720,964,924,211,050,490,834,741,684,000 a_{22}^{3} a_{40}^{8} b_{12} \\
& +7,826,499,497,633,136,164,923,512,183,750 a_{22} a_{40}^{12} b_{12} \\
& +4,469,135,964,829,014,341,045,756,192,882,688 a_{22}^{6} a_{40} b_{12}^{2} \\
& -60,534,442,756,814,886,093,718,953,699,609,600 a_{22}^{4} a_{40}^{5} b_{12}^{2} \\
& -4,533,006,178,962,510,464,756,656,493,703,000 a_{22}^{2} a_{40}^{9} b_{12}^{2} \\
& +4,340,383,679,701,089,115,056,480,723,750 a_{40}^{13} b_{12}^{2} \\
& +28,237,918,683,246,180,995,584,983,497,637,888 a_{22}^{5} a_{40}^{2} b_{12}^{3} \\
& -154,765,499,379,216,156,315,309,044,374,560,000 a_{22}^{3} a_{40}^{6} b_{12}^{3} \\
& -4,995,407,878,226,678,735,912,107,001,119,500 a_{22} a_{40}^{10} b_{12}^{3} \\
& +95,810,653,920,815,635,406,440,880,887,398,400 a_{22}^{4} a_{40}^{3} b_{12}^{4} \\
& -218,742,243,627,424,283,250,241,241,440,195,200 a_{22}^{2} a_{40}^{7} b_{12}^{4} \\
& -2,045,744,749,175,487,458,544,170,912,128,500 a_{40}^{11} b_{12}^{4} \\
& -813,586,646,290,453,924,746,048,307,200 a_{22}^{5} b_{12}^{5} \\
& +189,555,525,106,594,928,420,150,364,255,006,720 a_{22}^{3} a_{40}^{4} b_{12}^{5}
\end{aligned}
$$




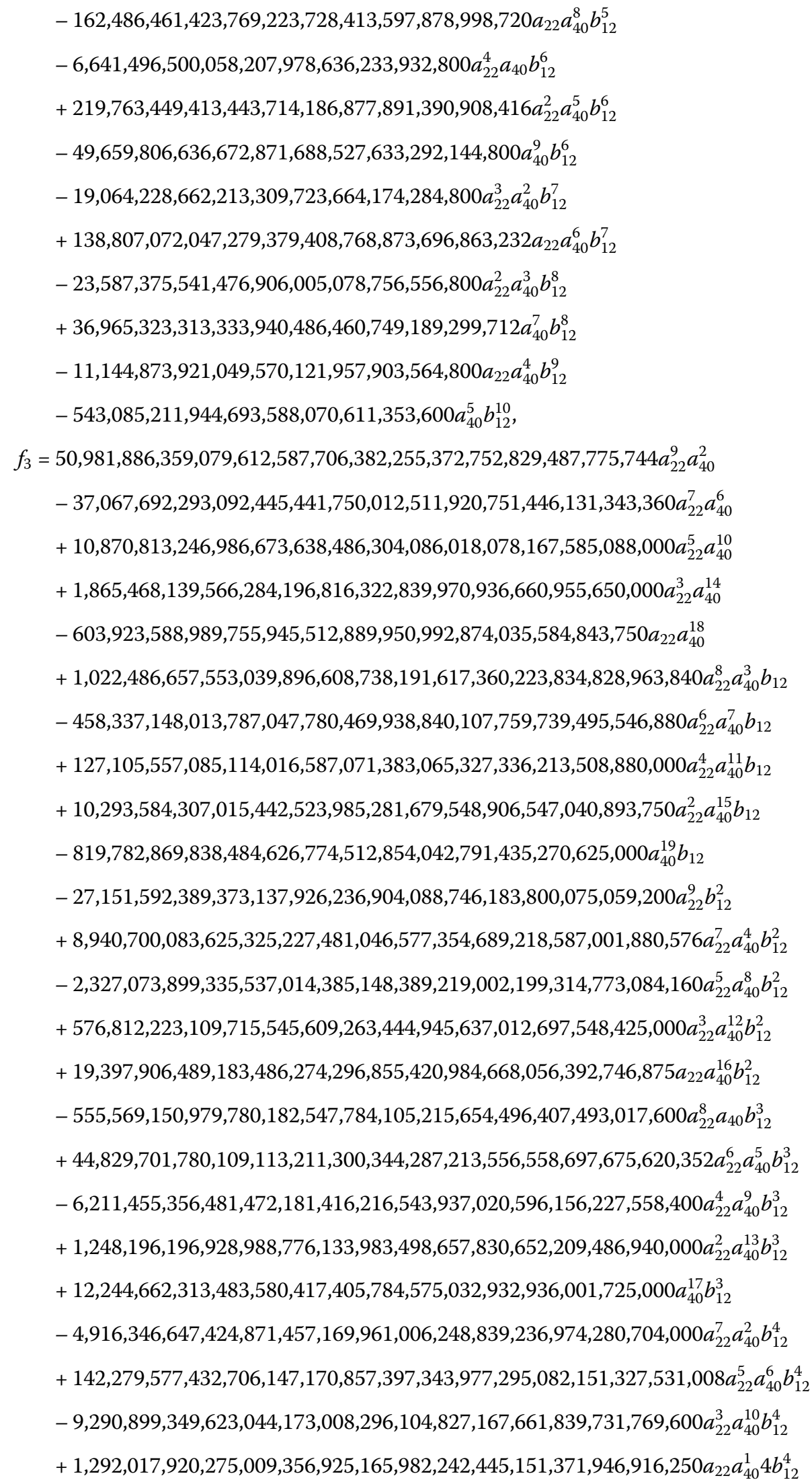




$$
\begin{aligned}
& \text { - 24,711,797,748,969,205,614,634,795,454,823,111,714,629,117,542,400a } a_{22}^{6} a_{40}^{3} b_{12}^{5} \\
& +296,723,701,499,161,287,704,516,622,860,637,906,314,000,798,846,976 a_{22}^{4} a_{40}^{7} b_{12}^{5} \\
& -7,598,375,984,298,187,054,214,113,602,180,794,604,878,607,085,440 a_{22}^{2} a_{40}^{11} b_{12}^{5} \\
& +515,403,389,037,848,152,893,742,601,009,301,446,964,737,813,250 a_{40}^{15} b_{12}^{5} \\
& +89,151,402,261,964,717,348,593,902,889,295,518,105,600,000 a_{22}^{7} b_{12}^{6} \\
& \text { - 77,871,378,889,486,757,955,374,362,879,501,321,944,798,907,596,800a } a_{22}^{5} a_{40}^{4} b_{12}^{6} \\
& +406,912,841,296,344,786,726,551,685,152,696,660,334,511,488,737,280 a_{22}^{3} a_{40}^{8} b_{12}^{6} \\
& -2,959,508,856,221,408,195,975,754,548,025,003,389,634,647,181,120 a_{22} a_{40}^{1} 2 b_{12}^{6} \\
& +1,212,459,455,348,222,745,203,216,678,389,937,329,930,240,000 a_{22}^{6} a_{40} b_{12}^{7} \\
& \text { - 159,874,291,856,994,698,343,598,397,136,909,959,783,750,585,548,800 } a_{22}^{4} a_{40}^{5} b_{12}^{7} \\
& +354,030,364,127,034,481,692,680,776,563,146,077,303,717,016,684,544 a_{22}^{2} a_{40}^{9} b_{12}^{7} \\
& \text { - 338,017,002,725,212,903,867,985,105,175,332,778,568,798,931,040 } a_{40}^{13} b_{12}^{7} \\
& +6,672,226,780,950,021,057,789,431,484,431,911,931,084,800,000 a_{22}^{5} a_{40}^{2} b_{12}^{8} \\
& \text { - 214,346,332,995,384,744,374,932,391,608,747,042,625,686,988,390,400 } a_{22}^{3} a_{40}^{6} b_{12}^{8} \\
& +177,421,389,360,763,691,749,439,937,259,376,019,693,424,578,797,568 a_{22} a_{40}^{10} b_{12}^{8} \\
& +19,201,252,172,312,399,449,409,674,516,768,771,434,086,400,000 a_{22}^{4} a_{40}^{3} b_{12}^{9} \\
& -181,375,047,489,804,263,451,726,975,105,980,803,657,328,001,024,000 a_{22}^{2} a_{40}^{7} b_{12}^{9} \\
& +39,043,027,707,216,401,602,019,440,086,182,542,407,958,295,162,112 a_{40}^{11} b_{12}^{9} \\
& +31,126,909,610,212,084,842,208,982,324,018,330,311,065,600,000 a_{22}^{3} a_{40}^{4} b_{12}^{10} \\
& -88,077,082,183,082,163,309,945,475,832,601,170,419,458,062,745,600 a_{22} a_{40}^{8} b_{12}^{10} \\
& +28,187,565,565,757,221,229,117,444,517,422,578,375,065,600,000 a_{22}^{2} a_{40}^{5} b_{12}^{11} \\
& -18,735,560,247,850,408,809,771,660,270,641,909,467,182,488,371,200 a_{40}^{9} b_{12}^{11} \\
& +12,916,473,356,332,131,362,657,092,325,292,496,398,581,760,000 a_{22} a_{40}^{6} b_{12}^{12} \\
& +2,179,454,745,027,373,896,621,572,921,424,926,638,080,000,000 a_{40}^{7} b_{12}^{13} \text {. }
\end{aligned}
$$




\section{References}

1. Qiu, J., Li, F.: Two kinds of bifurcation phenomena in a quartic system. Adv. Differ. Equ. 2015(1), 1 (2015)

2. Zhang, Z., Li, C.: On the number of limit cycles of a class of quadratic Hamiltonian systems under quadratic perturbations. Adv. Math. 26(5), 445-460 (1992)

3. Amelikin, B.B., Lukashivich, H.A., Sadovski, A.P.: Nonlinear Oscillations in Second Order Systems. BGY lenin.B. I. Press, Minsk (1992) (in Russian)

4. Han, M., Shu, C., Yang, J.: Polynomial Hamiltonian systems with a nilpotent critical point. Adv. Space Res. 46(4), 521-525 (2010)

5. Jiang, J., Zhang, J., Han, M.: Limit cycles for a class of quintic near-Hamiltonian systems near a nilpotent center. Int. J. Bifurc. Chaos 19(06), 2107-2113 (2009)

6. Yang, J., Han, M.: Limit cycle bifurcations of some Liénard systems with a nilpotent cusp. Int. J. Bifurc. Chaos 20(11), 3829-3839 (2010)

7. Han, M., Yang, J., Xiao, D.: Limit cycle bifurcations near a double homoclinic loop with a nilpotent saddle. Int. J. Bifurc. Chaos 22(08), $1250189(2012)$

8. An, Y., Han, M.: On the number of limit cycles near a homoclinic loop with a nilpotent singular point. J. Differ. Equ. 258(9), 3194-3247 (2015)

9. Giacomini, H., Gine, J., Llibre, J.: The problem of distinguishing between a center and a focus for nilpotent and degenerate analytic systems. J. Differ. Equ. 227(2), 406-426 (2006)

10. Algaba, A., García, C., Reyes, M.: The center problem for a family of systems of differential equations having a nilpotent singular point. J. Math. Anal. Appl. 340(1), 32-43 (2008)

11. Han, M., Sheng, L., Zhang, X.: Bifurcation theory for finitely smooth planar autonomous differential systems. J. Differ. Equ. 264, 3596-3618 (2018)

12. Han, M., Hou, X., Sheng, L., Wang, C.: Theory of rotated equations and applications to a population model. Discrete Contin. Dyn. Syst., Ser. A 34(8), 2171-2185 (2018)

13. Algaba, A., García, C., Gine, J.: Nilpotent centers via inverse integrating factors. Eur. J. Appl. Math. 27(5), 781-795 (2016)

14. Liu, Y., Li, J.: New study on the center problem and bifurcations of limit cycles for the Lyapunov system (I). Int. J. Bifurc. Chaos 19(11), 3791-3801 (2009)

15. Liu, Y., Li, J.: New study on the center problem and bifurcations of limit cycles for the Lyapunov system (II). Int. J. Bifurc Chaos 19(9), 3087-3099 (2009)

16. Liu, Y., Li, J.: Bifurcations of limit cycles and center problem for a class of cubic nilpotent system. Int. J. Bifurc. Chaos 20(08), 2579-2584 (2010)

17. Liu, Y., Li, F.: Double bifurcation of nilpotent focus. Int. J. Bifurc. Chaos 25(03), 1550036 (2015)

18. Liu, T., Wu, L., Li, F.: Analytic center of nilpotent critical points. Int. J. Bifurc. Chaos 22(08), 1250198 (2012)

19. Li, F.: Bifurcations of limit cycles in a quintic Lyapunov system with eleven parameters. Chaos Solitons Fractals 45(11), 1417-1422 (2012)

20. Li, F., Liu, Y., Li, H.: Center conditions and bifurcation of limit cycles at three-order nilpotent critical point in a septic Lyapunov system. Math. Comput. Simul. 81(12), 2595-2607 (2011)

21. Takens, F.: Singularities of vector fields. Publ. Math. IHES 43, 47-100 (1974)

22. Strozyna, E., Zoladek, H.: The analytic normal for the nilpotent singularity. J. Differ. Equ. 179, 479-537 (2002)

23. Moussu, R.: Symétrie et forme normale des centres et foyers degeneres. Ergod. Theory Dyn. Syst. 2, $241-251$ (1982)

24. Alvarez, M.J., Gasull, A.: Monodromy and stability for nilpotent critical points. Int. J. Bifurc. Chaos 15(4), 1253-1265 (2005)

25. Gamero, E., Freire, E., Ponce, E.: Normal forms for planar systems with nilpotent linear part, Bifurcation and chaos. In: Analysis, Algorithms, Applications, pp. 123-127. Birkhäuser, Basel (1991)

26. Alvarez, M.J., Gasull, A.: Generating limits cycles from a nilpotent critical point via normal forms. J. Math. Anal. Appl. $318,271-287(2006)$

27. Colak, I.E.: Hamiltonian linear type centers and nilpotent centers of linear plus cubic polynomial vector fields. Ph.D. thesis, Universitat Autònoma de Barcelona (2014)

28. Yu, P., Li, F.: Bifurcation of limit cycles in a cubic-order planar system around a nilpotent critical point. J. Math. Anal. Appl. 453(2), 645-667 (2017)

29. Bautin, N.: On the number of limit cycles which appear with the variation of coefficients from an equilibrium position of focus or center type. Math. Sb. (N.S.) 30, 181-196 (1952)

30. Zoldek, H.: Eleven small limit cycles in a cubic vector field. Nonlinearity 8, 843-860 (1995)

31. Christopher, C.: Estimating limit cycle bifurcations from centers. In: Differential Equations with Symbolic Computation, pp. 23-35. Birkhäuser, Basel (2005)

32. Gine, J.: Higher order limit cycle bifurcations from non-degenerate centers. Appl. Math. Comput. 218(17), 8853-8860 (2012) 